\title{
Transanal endoscopic microsurgery: is robotics the way to go?
}

\author{
A. Arezzo ${ }^{1} \cdot$ G. Gagliardi ${ }^{2}$ \\ Published online: 31 August 2021 \\ c) Springer Nature Switzerland AG 2021
}

The article by Atallah et al. [1] in this month's issue illustrates a novel robotic device to perform transanal surgery. The authors are at the forefront of innovations in transanal surgery. They introduced the concept of transanal minimally invasive surgery (TAMIS) in 2009, which attracted attention to this relatively old frontier of colorectal surgery. The development of transanal endoscopic microsurgery (TEM) had always been limited by the intrinsic difficulty of the technique introduced by Buess in 1983. Although TAMIS failed to prove any advantage in terms of efficacy [2], it attracted the interest of inventors who understood the potential of an easier way to remove early lesions of the large bowel transanally. The growing interest was fuelled by the increasing adoption of screening programs for colorectal cancer in western countries, with an increasing incidence of detection of lesions that were large but potentially removable with curative intent by adequate and oncological appropriate local excision.

How to achieve this by means of flexible endoscopy, either endoscopic mucosal resection (EMR) by snare or endoscopic submucosal dissection (ESD) by knife, is still a matter of debate. Despite several undoubted advances, flexible endoscopy has proved to be either too simplistic to solve the problem effectively (EMR) or too technically complicated to obtain both reliable and repeatable results and clinicians' interest in pursuing this technique in the western world (ESD).

Therefore, it is necessary to investigate new possibilities of visual screening and laboratory and local removal techniques that are easily reproducible with a short learning curve and provide reliable results in oncological terms. This would serve to limit the use of radical completion surgery

$\triangle$ A. Arezzo

alberto.arezzo@unito.it

1 Department of Surgical Sciences, University of Turin, c.so Dogliotti 14, 10126 Turin, Italy

2 Department of Surgery, University of Illinois College of Medicine at Chicago, Chicago, IL, USA in all cases, currently numerous, in which traditional techniques have not been able to remove "early" lesions with low risk of residual disease or recurrence.

It goes without saying that this involves the use of a "surgical" technique that allows the manipulation of the tissues so that an excision can be performed with the correct lateral and deep margins, which is lacking today in flexible endoscopy. Although TAMIS has shown it is possible to reduce the diameter of the instrumentation to be inserted transanally compared to the obsolete TEM, it has solved neither the problem of the upper limit reached, i.e., about $15 \mathrm{~cm}$ from the anal verge, nor the dexterity required to minimize the risks of incomplete excision.

The introduction of robotic technology is a significant step forward in this direction. Atallah's article reports a very short execution time, especially of the excisional phase, given that the suture, useful in case of perforation, does not always seem to be necessary. This is undoubtedly due to the possibility of having tissue manipulation during dissection. Evidently, robotics adds to transanal surgery the advantages of excellent ergonomics, tremor elimination, motion scaling, and multiple degrees of freedom. All the above characteristics are highly desirable when working in a confined space. Here instruments are often conflicting, and the right position to approach to the target not always obtainable, which can hamper both the operative flow and surgical precision.

Atallah and colleagues report an initial experience on the porcine colon model, with mock lesions measuring 2.5 to $3.5 \mathrm{~cm}$ excised by submucosal dissection. As reported by the authors, the system used is only partially robotic, as the forward and backward movement of the entire system depended on the assistant at the table. Robotics governed the two instruments for tissue manipulation and dissection by means of two standard Delta-robots man-machine interfaces (Fig. 1). The two flexible robotic instruments provide 7 degrees of freedom, each controlled by the surgeon's left and right hand, respectively. Indeed, this is a compromise that seems acceptable, compared to other solutions currently available or under development. Provided with a separate channel for a standard 5-mm endoscope to allow vision, the 
Fig. 1 The Colubriscope. The Colubriscope can translate forward and backward, upward and downward (arrows) as well as providing rotation along its long axis. The two flexible robotic instruments provide 7 degrees of freedom, each controlled by the surgeon's left and right hand, respectively

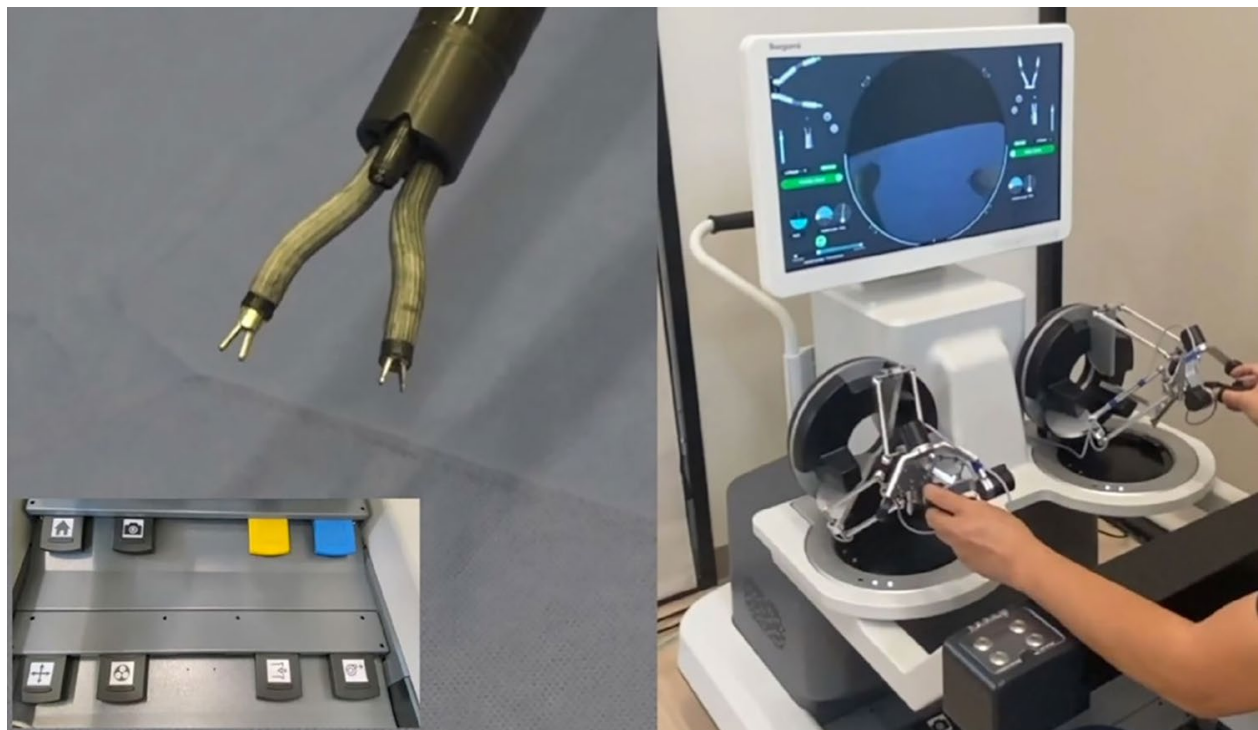

system allows for two articulated arms and it is possible to foresee the release of a longer device for applications above the rectosigmoid junction. The success of this platform will depend on the flexibility of the system and the capacity to approach the target lesion always in the correct position, which ideally should be at 6 o'clock of the visual field.

This approach is opposite to the approach chosen by Medrobotics Flex ${ }^{\circledR}$ Robot (Medrobotics, Raynham, MA, USA) [3]. Here the two arms are just mechanical, flexible to slide along the shaft of the endoscope, offering 7 degrees of freedom at the tip for an adequate approach to the target lesion. This also allows an easy exchange of the instruments on both arms, including dissecting, coagulation, and suturing tools. Robotics governs the steerable endoscope itself, which advances inside the lumen of the bowel, ignoring gravity.
The robotic scope is composed of inner and outer mechanisms, the surgeon advances and steers the outer mechanism and then the inner mechanism follows creating a stable surgical platform from which to operate (Fig. 2). The Flex ${ }^{\circledR}$ Robotic System is geared up with a haptic controller, joystick type, which can be moved in space in three dimensions. Theoretically, this system may also overtake the current limits of transanal endoscopic surgery and navigate above the rectosigmoid junction, but the current version is limited to $17 \mathrm{~cm}$. Longer and thinner versions are under development.

The more complex but, at the same time, more sophisticated approach, is undoubtedly the one chosen by Intuitive Surgical (Intuitive Surgical, Inc., Sunnyvale, CA, USA). Here three flexible instruments plus the arm holding a vision sensor are all governed robotically by the operator, who also
Fig. 2 The Medrobotics Flex® Robot. The Medrobotics Flex® Robot has two mechanical arms, flexible to slide along the shaft of the endoscope, offering 7 degrees of freedom at the tip. Robotics governs the steerable endoscope itself, which is composed of inner and outer mechanisms. The surgeon advances and steers the outer mechanism and then the inner mechanism follows creating a stable surgical platform from which to operate inside the lumen ignoring gravity

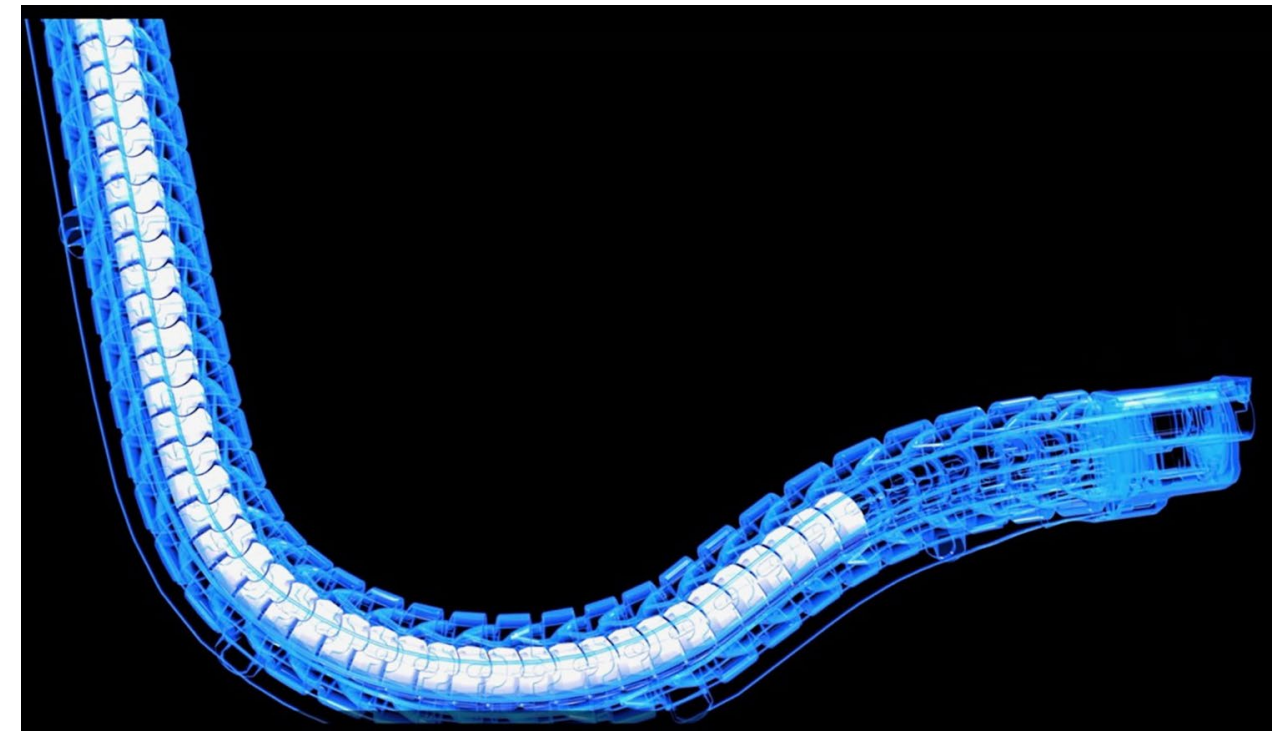


Fig. 3 The da Vinci SP. The da Vinci SP delivers an articulating 3D HD camera, along with three fully articulating instruments, with 7 degrees of freedom, through just one 25-mm cannula

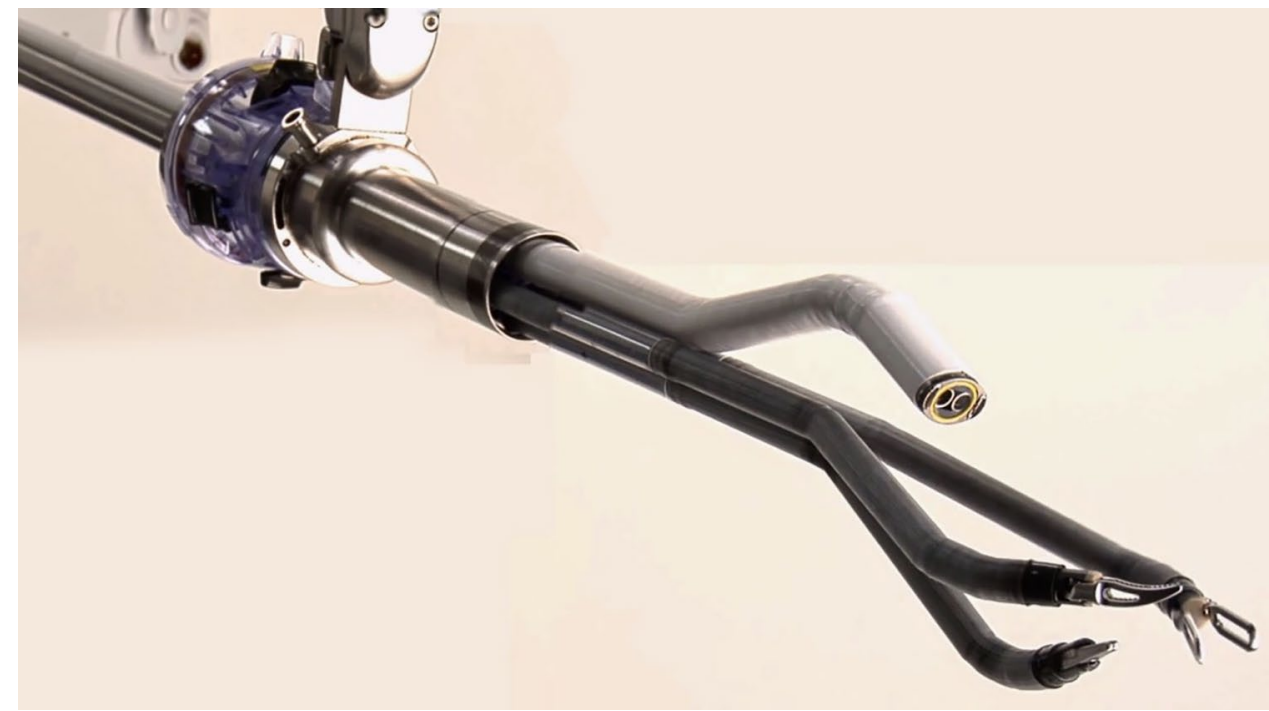

controls the insertion of the system inside the rectal cavity, with 7 degrees of freedom (Fig. 3). Nevertheless, the system is held by a relatively rigid shaft, which allows its use through the abdominal wall for single port applications for which it was conceived. Despite the excellent initial experience reported in this journal $[4,5]$, concerns remain about the ability to triangulate (i.e., exert traction and countertraction) in a small space such as the rectal cavity and the potential development of the system to navigate upwards above the rectum in future. Nevertheless, this certainly represents the only fully robotic system allowing transanal endoscopic surgery so far, which it does in an outstanding manner.

Someone might question the added benefit of the robot, given the financial implications. The evolution of the use of robotics in surgery demonstrates that in the presence of a clear clinical benefit the issue of cost becomes secondary, at least in western countries. Here robotic technology has the advantage, due to miniaturization, of offering a real surgical environment otherwise inaccessible. Therefore, clinical benefits are even more likely to be proven compared to other current applications. How sophisticated this should be will be ascertained by preclinical and clinical applications.

Similar systems should permit a higher number of patients to benefit from the advantages of minimally invasive endoluminal surgery [9]. Although the present series represents a work in progress, the reliability to achieve better results in en-bloc $\mathrm{R} 0$ resections and, consequently, recurrence rate, compared to standard flexible endoscopy, has to be proven [10].

Research activity should be focused not only on conceiving longer devices to reach lesions above the recto-sigmoid junction. More important is the required progress in precision surgery, increased dexterity and user-friendliness, to significantly reduce incomplete resections and perioperative complications. Flexible robotic technologies are expected to improve the overall performance of difficult dissection inside the gastrointestinal lumen. Further progress is mandatory to allow a surgical method primarily based on tissue manipulation, traction, and counter traction to achieve this.

Acknowledgements This study was supported by Ministero dell'Istruzione, dell'Università e della Ricerca (MIUR) under the programme "Dipartimenti di Eccellenza ex L.232/2016" to the Dept. of Surgical Sciences, University of Torino.

\section{Declarations}

Conflict of interest Dr. Alberto Arezzo has served as a consultant for Medrobotics (Raynham, MA, USA).

Ethical approval This article does not contain any studies with human participants performed by any of the authors.

Informed consent For this type of study, formal consent is not required.

\section{References}

1. Atallah S, Sanchez A, Bianchi E, Larach SW (2021) Envisioning the future of colorectal surgery: preclinical assessment and detailed description of an endoluminal robotic system (ColubrisMX ELS). Tech Coloproctol. https://doi.org/10.1007/s10151021-02481-0( (Epub ahead of print) $)$

2. Van den Eynde F, Jaekers J, Fieuws S, D'Hoore AM, Wolthuis AM (2019) TAMIS is a valuable alternative to TEM for resection of intraluminal rectal tumors. Tech Coloproctol 23(2):161-166

3. Arezzo A, Forcignanò E, Morino M (2020) Robotic endoscopic submucosal dissection and full-thickness excision for laterally spreading tumors of the rectum. Minim Invasive Ther Allied Technol. https://doi.org/10.1080/13645706.2020.1826972 ((Epub ahead of print))

4. Liu S, Kelley SR, Behm KT (2021) Single-port robotic transanal minimally invasive surgery (SPR-TAMIS) approach to local excision of rectal tumors. Tech Coloproctol 25(2):229-234. https:// 
doi.org/10.1007/s10151-020-02286-7 (Epub 2020 Jul 6 PMID: 32632706)

5. Marks JH, Kunkel E, Salem JF, Martin C, Anderson B, Agarwal S (2021) First clinical experience with single-port robotic transanal minimally invasive surgery (SP rTAMIS) for benign rectal neoplasms. Tech Coloproctol 25(1):117-124

6. Arezzo A, Mintz Y, Allaix ME, Arolfo S, Bonino M, Gerboni G, Brancadoro M, Cianchetti M, Menciassi A, Wurdemann H, Noh Y, Althoefer K, Fras J, Glowka J, Nawrat Z, Cassidy G, Walker R, Morino M (2017) Total mesorectal excision using a soft and flexible robotic arm: a feasibility study in cadaver models. Surg Endosc 31(1):264-273

7. Verra M, Firrincieli A, Chiurazzi M, Mariani A, Lo Secco G, Forcignanò E, Koulaouzidis A, Menciassi A, Dario P, Ciuti G, Arezzo A (2020) Robotic-Assisted Colonoscopy Platform with a Magnetically-Actuated Soft-Tethered Capsule. Cancers (Basel) 12(9):2485

8. Martin JW, Scaglioni B, Norton JC, Subramanian V, Arezzo A, Obstein KL, Valdastri P (2020) Enabling the future of colonoscopy with intelligent and autonomous magnetic manipulation. Nat Mach Intell. 2(10):595-606. https://doi.org/10.1038/ s42256-020-00231-9 (Epub 2020 Oct 12. PMID: 33089071; PMCID: PMC7571595)

9. de Moura DTH, Aihara H, Jirapinyo P, Farias G, Hathorn KE, Bazarbashi A et al (2019) Robot-assisted endoscopic submucosal dissection versus conventional ESD for colorectal lesions: outcomes of a randomized pilot study in endoscopists without prior ESD experience. Gastrointest Endosc 90:290-298

10. Morino M, Forcignanò E, Arezzo A (2021) Early clinical adoption of a flexible robotic endoscope for local excision of rectal lesions. Br J Surg 10:znab193. https://doi.org/10.1093/bjs/znab193 (Epub ahead of print)

Publisher's Note Springer Nature remains neutral with regard to jurisdictional claims in published maps and institutional affiliations. 